\title{
Ansiedad en el paciente prequirúrgico: un problema que nos afecta a todos
}

\author{
Andrea Quintero ${ }^{1}$, Diana A. Yasnó ${ }^{2}$, Olga L. Riveros ${ }^{2}$, Jeisson Castillo ${ }^{2}$, Bernardo A. Borráez ${ }^{2,3}$
}

Palabras clave: ansiedad; cirugía general; colecistectomía laparoscópica; medicina de la conducta; psicología.

\section{Resumen}

Introducción. La ansiedad es el estado mental que conduce al ser humano a experimentar reacciones de incomodidad, angustia, miedo, preocupación o excitación. Los procesos quirúrgicos desencadenan una serie de reacciones mentales, emocionales y físicas que pueden aumentar el grado de ansiedad, llegando al punto de influir en el proceso de recuperación de los pacientes que son sometidos a cirugías.

Los objetivos de nuestro estudio fueron determinar el estado de ansiedad de los pacientes llevados a colecistectomía en un servicio de cirugía y determinar la asociación entre el grado de ansiedad y el sexo, la presencia de antecedentes quirúrgicos y el tiempo de hospitalización previo al procedimiento quirúrgico.

Materiales y métodos. Se aplicó la escala de ansiedad de Beck en pacientes que iban a ser sometidos a

1 Departamento de Psicología, Fundación Clínica Shaio, Bogotá, D.C., Colombia

2 Departamento de Cirugía General, Fundación Clínica Shaio, Bogotá, D.C., Colombia.

3 Departamento de Cirugía Gastrointestinal y Endoscopia Digestiva, Instituto Nacional de Cancerología, Bogotá, D.C. Colombia

Fecha de recibido: 26 de octubre de 2016

Fecha de aprobación: 7 de marzo de 2017

Citar como: Quintero A, Yasnó DA, Riveros OL, Castillo J, Borráez BA. Ansiedad en el paciente prequirúrgico: un problema que nos afecta a todos. Rev Colomb Cir. 2017; 32:115-20. colecistectomía laparoscópica entre abril y julio de 2016. En estos pacientes se utilizaron técnicas de intervención y estabilización emocional. Se compararon los niveles de ansiedad en términos de sexo, presencia o ausencia de antecedentes quirúrgicos, y realización de la intervención antes o después de las primeras 24 horas del ingreso.

Resultados. Se incluyeron 21 pacientes intervenidos quirúrgicamente, con un rango de edad entre los 23 y los 93 años, en quienes se practicó colecistectomía laparoscópica. Todos los pacientes intervenidos presentaron algún grado de ansiedad: leve en 7 , moderada en 11 y grave en 3. Los hombres presentaron con mayor frecuencia niveles altos de ansiedad ( $p=0,3458)$. Los pacientes que presentaron un antecedente quirúrgico previo al procedimiento tuvieron menores grados de ansiedad $(p=0,0071)$. Nueve pacientes cursaron con procesos de adaptación hospitalaria y 12 fueron intervenidos el mismo día de la hospitalización, sin diferencia estadísticamente significativa en el grado de ansiedad.

Conclusiones. Los resultados de este estudio muestran que no se encuentra asociación entre el grado de ansiedad y el sexo, o el tiempo de hospitalización previo a la realización del procedimiento quirúrgico, mientras que la presencia de antecedentes quirúrgicos genera un menor grado de ansiedad en quienes van a ser intervenidos. La intervención psicológica prequirúrgica y posquirúrgica podría permitir identificar factores que generan ansiedad, mejorar la adaptación antes y después del procedimiento, y generar espacios óptimos para complementar información y aclarar dudas sobre el procedimiento. 


\section{Introducción}

La ansiedad se define como el estado o condición mental en que el ser humano experimenta reacciones de incomodidad, angustia, miedo y preocupación; hace parte de la reacción humana a situaciones de amenaza, conocida como la reacción psicológica a factores de estrés, la cual tiene componentes psicológicos y fisiológicos ${ }^{1,2}$.

La ansiedad cumple una función adaptativa que se considera una reacción habitual ante las demandas del ambiente. Sin embargo, en ocasiones, tiende a superar las capacidades propias y a generar descontrol frente a alguna situación, ya sea por su intensidad, difícil manejo o, incluso, por carencia de recursos internos que permitan ampliar el repertorio de reacción, lo cual genera un manejo inadecuado de las estrategias de afrontamiento $^{2,3}$.

Los procesos quirúrgicos desencadenan una serie de reacciones de manera preventiva (mentales, emocionales y físicas), antes, durante y después del procedimiento, que aumentan los niveles de ansiedad y podrían, incluso, influir en el proceso de recuperación. La ansiedad puede provocar una reacción fisiológica (cambios en el metabolismo - glucosa-, activación del sistema nervioso simpático -aumento de la presión arterial y taquicardia-, y activación del sistema endocrino e inmunológico), y una reacción muy similar a la generada en episodios clásicos de estrés mental ${ }^{3-6}$.

El objetivo del presente estudio fue identificar los factores que influyen en el estado de ansiedad de los pacientes intervenidos quirúrgicamente en un procedimiento frecuente (colecistectomía por laparoscopia), y presentar nuestra experiencia con la implementación de una herramienta para la identificación y la clasificación de la ansiedad, y con base en ello, aplicar técnicas de intervención y estabilización en busca de una mejor adaptación, estancia y recuperación.

\section{Materiales y métodos}

Se aplicó la escala de ansiedad de Beck (Beck Anxiety Inventory) en 21 pacientes que iban a ser sometidos a colecistectomía laparoscópica entre el $1^{\circ}$ de abril y el 31 de julio de 2016. Se compararon los niveles de ansiedad según el sexo, la presencia de antecedentes quirúrgicos y la realización del procedimiento quirúrgico antes o después de las primeras 24 horas del ingreso.
Escala de ansiedad de Beck. Es una escala validada de medición de la ansiedad, utilizada como instrumento de evaluación de la ansiedad clínica, debido a que discrimina mejor entre ansiedad y depresión, y muestra índices psicométricos adecuados para medir la presencia y la gravedad de la sintomatología de ansiedad ${ }^{5}$.

En la escala de ansiedad de Beck se suman todos los puntajes obtenidos en cada uno de los 21 ítems, y el grado de ansiedad se califica de 0 a 3: desde 0 , cuando está ausente, hasta 3 , cuando es grave. La ansiedad se clasifica en cuatro rangos previamente establecidos, de la siguiente manera: mínima, de 0 a 7 ; leve, de 8 a 15 , moderada, de 16 a 25 , y grave, de 26 a $63{ }^{5}$.

Intervención. En estos pacientes se utilizaron técnicas de intervención y estabilización emocional, asociadas a psicoeducación, respiración diafragmática, habilidades de tolerancia al malestar y potenciar estrategias de afrontamiento.

Se llevaron a cabo en dos sesiones. La primera sesión, la de intervención, duró 45 minutos: 15 minutos de aplicación del inventario para identificar niveles de ansiedad y 30 minutos de intervención según el estado del paciente durante valoración.

La estrategia empleada en esta sesión consta de cuatro pasos básicos:

1. Psicoeducación frente al procedimiento y posibles cambios conductuales y emocionales asociados.

2. Entrenamiento en técnicas de respiración diafragmática.

3. Habilidades de tolerancia al malestar.

4. Evaluación y potenciación de las estrategias de afrontamiento.

Después del procedimiento quirúrgico, se realizó una segunda sesión, la de seguimiento, de 30 minutos, aproximadamente. Esta sesión tiene como propósito principal evaluar una intervención previa, por medio de la retroalimentación y el soporte generado por el discurso del paciente, lo cual permite cerrar de manera adecuada el ciclo terapéutico. Se brinda espacio para ventilar y elaborar las emociones asociadas a las experiencias antes y después de la intervención quirúrgica. 
El análisis estadístico se hizo con SPSS $16.0^{\circledR}$ para MAC OSX, usando la prueba de ji al cuadrado y la prueba exacta de Fisher. Los datos se expresaron en medias y desviaciones estándar. Un valor de $\mathrm{p}$ de 0,05 se consideró estadísticamente significativo.

\section{Resultados}

Se les practicó colecistectomía laparoscópica a 21 pacientes, entre el $1^{\circ}$ de agosto y el 31 de diciembre de 2016. Trece pacientes eran de sexo masculino, con un rango de edad entre los 27 y los 85 años (media $=55$ años), y 8 pacientes de sexo femenino, con un rango de edad entre 23 y 93 años (media=58 años).

Todos los pacientes intervenidos presentaron algún grado de ansiedad: en 7 ( 3 hombres y 4 mujeres), fue leve; en 11 ( 8 hombres y 3 mujeres), moderada, y en 3 (2 hombres y 1 mujer), grave; ninguno presentó ansiedad mínima. A pesar de que un número mayor de pacientes masculinos presentó un mayor porcentaje de ansiedad moderada y grave, no se encontró diferencia estadísticamente significativa según el sexo (tabla 1).

TABLA 1.

Grado de ansiedad según el sexo en pacientes sometidos a colecistectomía por laparoscopia

\begin{tabular}{lccc}
\hline $\begin{array}{c}\text { Grado de } \\
\text { ansiedad }\end{array}$ & $\begin{array}{c}\text { Hombres } \\
(\mathbf{n = 1 3 )} \\
\mathbf{n}(\mathbf{\%})\end{array}$ & $\begin{array}{c}\text { Mujeres } \\
(\mathbf{n = 8 )} \\
\mathbf{n}(\mathbf{\%})\end{array}$ & $\mathbf{p}$ \\
\hline Mínima & $0(0)$ & $0(0)$ & 0 \\
\hline Leve & $3(23,1)$ & $4(50)$ & 0,3458 \\
\hline Moderada & $8(61,5)$ & $3(37,5)$ & 0,3870 \\
\hline Grave & $2(15,4)$ & $1(12,5)$ & 1 \\
\hline
\end{tabular}

De los 21 pacientes, 12 tenían antecedentes quirúrgicos, como prostatectomía, apendicectomía, cirugía cardiaca o cesárea. Los grados altos de ansiedad, moderada y grave, fueron menos frecuentes en aquellos con antecedente quirúrgico: 5 con este antecedente y $9 \sin$ el mismo ( $p=0,0071)$ (figura 1).

Nueve de los 21 pacientes cursaron con procesos de adaptación hospitalaria (intervenidos quirúrgicamente después de 24 horas de su ingreso a la institución) y 12 fueron intervenidos el mismo día de la hospitalización.
De los que pasaron por un proceso de adaptación hospitalaria, en tres, la ansiedad fue mínima o leve, y en seis, moderada o grave; de los pacientes intervenidos en las primeras 24 horas después del ingreso, en cuatro, fue mínima o leve, y en ocho, moderada o grave. No se presentó diferencia estadísticamente significativa entre los dos grupos (figura 2).

Entre los pacientes con los mayores grados de ansiedad, hubo: dos hombres y una mujer con ansiedad grave, tres pacientes sin antecedentes quirúrgicos y tres que fueron operados en las primeras 24 horas después del ingreso.

En la intervención prequirúrgica, se encontró que la ansiedad se desencadena, según los pacientes, por “poca información del procedimiento, efectos adversos

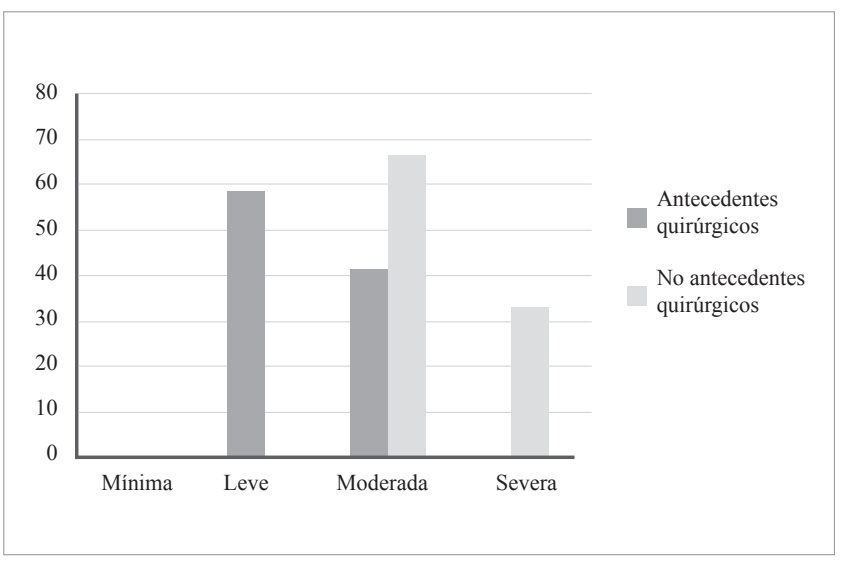

Figura 1. Grado de ansiedad según la presencia o ausencia de antecedentes quirúrgicos

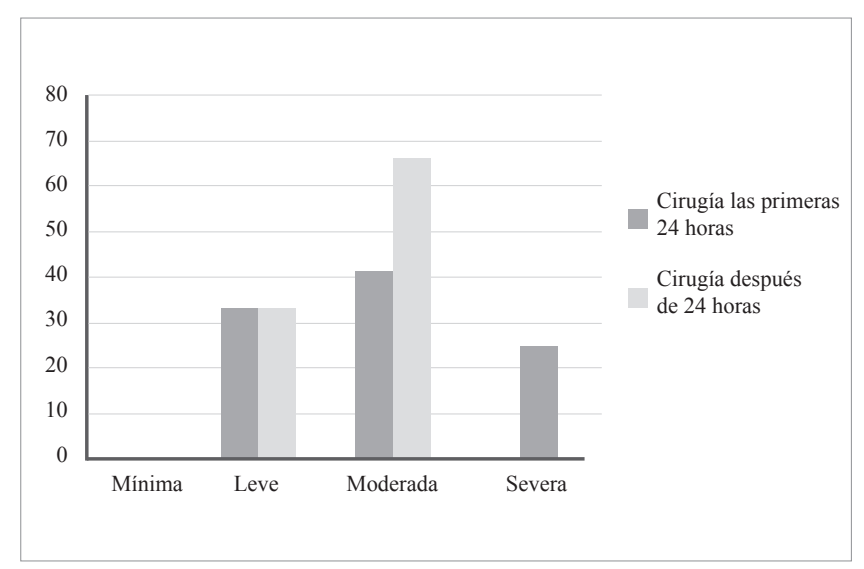

FIGURA 2. Grado de ansiedad según el tiempo transcurrido entre el ingreso y el procedimiento quirúrgico 
y consecuencias a raíz de la intervención”. Durante esta intervención, se respondieron respuestas simples sobre el procedimiento, sus riesgos y complicaciones.

\section{Discusión}

Los resultados de este estudio muestran que: a) los procedimientos quirúrgicos generan ansiedad, en su mayoría leve y moderada; b) no se encuentra asociación entre el grado de ansiedad y el sexo o el tiempo de hospitalización antes del procedimiento quirúrgico, mientras que, con la presencia de antecedentes quirúrgicos, se genera un menor grado de ansiedad; y c) las intervenciones psicológicas prequirúrgica y posquirúrgica permiten identificar factores que generan ansiedad, mejorar la adaptación antes y después de la cirugía, y generar un espacio óptimo para complementar la información y aclarar las dudas sobre el procedimiento.

La ansiedad es una reacción natural que experimenta el ser humano cuando se enfrenta a situaciones peligrosas, desconocidas o que impliquen alto riesgo. La reacción de ansiedad antes de cualquier intervención quirúrgica se puede considerar uno de los mayores problemas durante la preparación prequirúrgica; todos los pacientes en este estudio presentaron algún grado de ansiedad antes de la cirugía. Los altos niveles de ansiedad han demostrado influir negativamente en los procedimientos quirúrgicos, al generar cambios en el sistema inmunológico; se ha demostrado que la ansiedad preoperatoria se asocia con una recuperación más lenta y dolorosa, y con mayor cantidad de complicaciones ${ }^{1,7}$.

En el presente estudio se encontraron mayores grados de ansiedad en hombres, sin una diferencia estadísticamente significativa; estos resultados no concuerdan con los de Ay, et al., y Mavridou, et al., quienes encontraron mayores grados de ansiedad en las mujeres de un grupo de pacientes sometidos a colecistectomía y otros tipos de procedimientos. Esto podría explicarse por el bajo número de pacientes de la presente serie ${ }^{8,9}$.

No hubo una diferencia estadísticamente significativa relacionada con el acceso al proceso de adaptación hospitalaria, a pesar de encontrarse un porcentaje mayor de pacientes con ansiedad importante entre quienes fueron operados en las primeras 24 horas. Los hallazgos de Cárdenas, et al., y Pereira, et al., encontraron niveles de ansiedad similares en pacientes sometidos a cirugía ambulatoria, lo que evidencia que todo proceso quirúrgico conlleva algún grado de ansiedad ${ }^{1,10}$.

La ansiedad fue menor, con una diferencia estadísticamente significativa, en quienes tenían antecedentes quirúrgicos, lo cual concuerda con los hallazgos de Mavridou, et al., y Ay, et al.; probablemente, esto se asocia con una experiencia ya conocida y una educación previa sobre los eventos perioperatorios ${ }^{8,9}$.

Otros factores no evaluados en este estudio se han asociado con el grado de ansiedad, como el bajo nivel educativo y la edad (pacientes jóvenes); en parte, estos factores se asocian con el cuidado perioperatorio, la anestesia, el dolor posoperatorio y las secuelas ${ }^{8,9}$.

En este estudio, se brindó acompañamiento por parte del Departamento de Psicología, lo que permitió evidenciar que los pacientes, a pesar de ser informados del procedimiento, resolver sus dudas y firmar un consentimiento informado, refieren tener poca información sobre el procedimiento o requieren más. Durante la sesión de intervención, se aportó información adicional y se potenciaron las estrategias de afrontamiento, con el propósito de brindar un mejor manejo de la ansiedad antes de la cirugía. Aunque no fue un objetivo de este trabajo evaluar la utilidad de las intervenciones, se encontró que esta intervención permite ampliar la información recibida por los pacientes, con un aparente impacto favorable ${ }^{3,11-13}$.

\section{Conclusiones}

A pesar de reconocer que el presente estudio tiene limitaciones como el pequeño tamaño de la muestra, se puede concluir que los procedimientos quirúrgicos, como la colecistectomía laparoscópica, generan ansiedad, generalmente leve o moderada, y que hay una diferencia estadísticamente significativa según los antecedentes quirúrgicos, sin ninguna asociación con el sexo o el tiempo de hospitalización previo al procedimiento quirúrgico.

Además, consideramos que podría requerirse un acompañamiento psicológico con el complemento de una intervención breve, que permita un mejor afrontamiento del proceso quirúrgico y brindar información adicional sobre el procedimiento. 


\title{
Preoperative anxiety: a problem affecting all of us
}

\begin{abstract}
Background. Anxiety is a mental state that leads people to reactions of discomfort, anguish, fear, preoccupation or excitation. Surgical interventions trigger a series of mental, emotional and physical responses that can increase the levels of anxiety to the point of affecting the recovery of the patients following surgery.

The objective of this study was to determine the state of anxiety of patients undergoing cholecystectomy and the association of the level of anxiety with the patient's gender, the history of previous surgical interventions and the time of hospital stay previous to the scheduled procedure.

Patients and methods. Beck anxiety inventory scale was applied in 21 patients in whom laparoscopic cholecystectomy was performed in the period August 1st to December 31st, 2015. Interventional and emotional stabilization techniques were applied, and we compared the anxiety levels regarding gender, presence or absence of previous surgical interventions and the performance of the operation before or after 24 hour hospital admission.

Results. Twenty one patients undergoing laparoscopic cholecystectomy were included, with ages ranging from 23 to 93 years. Thirteen were male and eight female. All patients presented some degree of anxiety: mild 7, moderate 11, and severe 3. Higher levels of anxiety were more frequent in males, with no statistically significant difference (10 vs. 4 patients respectively, $p=0.3458$ ). Patients who had history of previous surgery exhibited lower levels of anxiety than the patients without such history $(p=0.0071)$. Nine patients had hospital stays longer than 24 hours before surgery and in 12 patients were operated on the same day of admission, with no statistically significant difference in the level of anxiety.

Conclusions. These results show that there was no association between the level of anxiety and gender, or the time of hospital stay prior to the operation, while the history of previous surgery is associated lower levels of anxiety. Pre and postoperative psychological intervention could identify factors that generate anxiety, improve the emotional adaptation before and after the procedure, and generate ample spaces to complement information and clear doubts regarding the surgical procedure.
\end{abstract}

Key words: Anxiety; general surgery; cholecystectomy, laparoscopic; behavioral medicine; psychology.

\section{Referencias}

1. Pereira L, Figueiredo-Braga M, Carvalho IP. Preoperative anxiety in ambulatory surgery: The impact of an empathic patient-centered approach on psychological and clinical outcomes. Patient Educ Couns. 2016;99:733-8.

2. Kipnis G, Tabak N, Koton S. Background music playback in the preoperative setting: Does it reduce the level of preoperative anxiety among candidates for elective surgery? J Perianesth Nurs. 2016;31:209-16.

3. Doñate M, Litago A, Monge Y, Martínez R. Preoperative aspects of information elated to patient anxiety scheduled for surgery. Enfermería Global. 2015;37:170-80.

4. Attias S, Keinan Boker L, Arnon Z, Ben-Arye E, Bar'am A, Sroka G. et al. Effectiveness of integrating individualized and generic complementary medicine treatments with standard care versus standard care alone for reducing preoperative anxiety. $\mathrm{J}$ Clin Anesth. 2016;29:54-64.
5. Sanz J. Recomendaciones para la utilización de la adaptación española del Inventario de Ansiedad de Beck (BAI) en la práctica clínica. Clínica y Salud. 2014;25:39-48.

6. Hernández Y. Hipertensión arterial perioperatoria: ¿Cuándo operar? Rev Col Anest. 2005;33:269-81.

7. Carapia A, Mejía G, Nacif L, Hernández N. Efecto de la intervención psicológica sobre la ansiedad preoperatoria. Rev Mex Anest. 2011;34:260-3.

8. Ay AA, Ulucanlar H, Ay A, Ozden M. Risk factors for perioperative anxiety in laparoscopic surgery. JSLS. 2014;18. pii: e2014.00159.

9. Mavridou P, Dimitriou V, Manataki A, Arnaoutoglou E, Papadopoulos G. Patient's anxiety and fear of anesthesia: Effect of gender, age, education, and previous experience of anesthesia. A survey of 400 patients. J Anesth. 2013;27:104-8. 
10. Cárdenas A, Quiroga C, Restrepo M, Cortés D. Histerectomía y ansiedad: estudio comparativo entre dos tipos de preparación psicológica prequirúrgica. Rev Col Obst Gin. 2005; 56: 209-15.

11. Díez-Álvarez E, Arrospide A, Mar J, Álvarez U, Belaustegi A, Lizaur B, et al. Effectiveness of pre-operative education in reducing anxiety in surgical patients. Enferm Clin. 2012;22:18-26.

12. Castillero Y. Intervención psicológica en cirugía cardíaca. Av Psicol Latinoam. 2007;25:52-63.
13. Aust H, Rüsch D, Schuster M, Sturm T, Brehm F, Nestoriuc Y. Coping strategies in anxious surgical patients. BMC Health Serv Res. 2016;16:1-10.

Correspondencia: Bernardo A. Borráez, MD Correo electrónico: bborraez@hotmail.com Bogotá, D.C. 